\title{
Some applications of differential subordination to certain subclass of p-valent meromorphic functions involving convolution
}

\author{
T. M. Seoudy \\ Fayoum University \\ Faculty of Science \\ Department of Mathematics \\ Fayoum 63514, Egypt \\ email: tms00@fayoum.edu.eg
}

\author{
M. K. Aouf \\ Mansoura University \\ Faculty of Science \\ Department of Mathematics \\ Mansoura 35516, Egypt \\ email: mkaouf127@yahoo.com
}

\begin{abstract}
By using the principle of differential subordination, we introduce subclass of $p$-valent meromorphic functions involving convolution and investigate various properties for this subclass. We also indicate relevant connections of the various results presented in this paper with the obtained results in earlier works.
\end{abstract}

\section{Introduction}

For any integer $m>-p$, let $\Sigma_{p, m}$ denote the class of all meromorphic functions $f$ of the form:

$$
f(z)=z^{-p}+\sum_{k=m}^{\infty} a_{k} z^{k} \quad(p \in \mathbb{N}=\{1,2, \ldots\}),
$$

which are analytic and $p$-valent in the punctured disc $\mathrm{U}^{*}=\{z \in \mathbb{C}: 0<|z|<$ $1\}=U \backslash\{0\}$. For convenience, we write $\Sigma_{p,-p+1}=\Sigma_{p}$. If $f$ and $g$ are analytic in 
$\mathrm{U}$, we say that $\mathrm{f}$ is subordinate to $\mathrm{g}$, written symbolically as, $\mathrm{f} \prec \mathrm{g}$ or $\mathrm{f}(\mathrm{z}) \prec$ $g(z)$, if there exists a Schwarz function $w$, which (by definition) is analytic in $\mathrm{U}$ with $w(0)=0$ and $|w(z)|<1(z \in \mathrm{U})$ such that $\mathrm{f}(z)=\mathrm{g}(w(z))(z \in \mathrm{U})$. In particular, if the function $\mathrm{g}$ is univalent in $\mathrm{U}$, we have the equivalence (see [10] and [11]):

$$
\mathrm{f}(z) \prec \mathrm{g}(z) \Leftrightarrow \mathrm{f}(0)=\mathrm{g}(0) \text { and } \mathrm{f}(\mathrm{U}) \subset \mathrm{g}(\mathrm{U}) .
$$

For functions $f \in \Sigma_{p, m}$, given by (1), and $g \in \Sigma_{p, m}$ defined by

$$
g(z)=z^{-p}+\sum_{k=m}^{\infty} b_{k} z^{k}(m>-p ; p \in \mathbb{N}),
$$

then the Hadamard product (or convolution) of $f$ and $g$ is given by

$$
(f * g)=z^{-p}+\sum_{k=m}^{\infty} a_{k} b_{k} z^{k}=(g * f)(z) \quad(m>-p ; p \in \mathbb{N}) .
$$

For complex parameters

$$
\alpha_{1}, \ldots, \alpha_{q} \text { and } \beta_{1}, \ldots, \beta_{s}\left(\beta_{j} \notin \mathbb{Z}_{0}^{-}=\{0,-1,-2, \ldots\} ; j=1,2, \ldots, s\right),
$$

we now define the generalized hypergeometric function ${ }_{q} F_{s}\left(\alpha_{1}, \ldots, \alpha_{q}\right.$; $\left.\beta_{1}, \ldots, \beta_{s} ; z\right)$ by (see, for example, $[14$, p.19])

$$
\begin{gathered}
{ }_{\mathrm{q}} \mathrm{F}_{\mathrm{s}}\left(\alpha_{1}, \ldots, \alpha_{\mathrm{q}} ; \beta_{1}, \ldots, \beta_{s} ; z\right)=\sum_{\mathrm{k}=0}^{\infty} \frac{\left(\alpha_{1}\right)_{\mathrm{k}} \ldots\left(\alpha_{\mathrm{q}}\right)_{\mathrm{k}}}{\left(\beta_{1}\right)_{\mathrm{k}} \ldots\left(\beta_{\mathrm{s}}\right)_{\mathrm{k}}} \cdot \frac{z^{\mathrm{k}}}{\mathrm{k} !} \\
\left(\mathrm{q} \leq \mathrm{s}+1 ; \mathrm{q}, \mathrm{s} \in \mathbb{N}_{0}=\mathbb{N} \cup\{0\} ; z \in \mathrm{U}\right),
\end{gathered}
$$

where $(\theta)_{v}$ is the Pochhammer symbol defined, in terms of the Gamma function, by

$$
(\theta)_{v}=\frac{\Gamma(\theta+v)}{\Gamma(\theta)}= \begin{cases}1 & \left(v=0 ; \theta \in \mathbb{C}^{*}=\mathbb{C} \backslash\{0\}\right), \\ \theta(\theta-1) \ldots(\theta+\nu-1) & (\nu \in \mathbb{N} ; \theta \in \mathbb{C}) .\end{cases}
$$

Corresponding to the function $h_{p}\left(\alpha_{1}, \ldots, \alpha_{q} ; \beta_{1}, \ldots, \beta_{s} ; z\right)$, defined by

$$
h_{p}\left(\alpha_{1}, \ldots, \alpha_{q} ; \beta_{1}, \ldots, \beta_{s} ; z\right)=z^{-p}{ }_{q} F_{s}\left(\alpha_{1}, \ldots, \alpha_{q} ; \beta_{1}, \ldots, \beta_{s} ; z\right),
$$

we consider a linear operator

$$
H_{p}\left(\alpha_{1}, \ldots, \alpha_{q} ; \beta_{1}, \ldots, \beta_{s} ; z\right): \Sigma_{p} \rightarrow \Sigma_{p},
$$


which is defined by the following Hadamard product (or convolution):

$$
H_{p}\left(\alpha_{1}, \ldots, \alpha_{q} ; \beta_{1}, \ldots, \beta_{s}\right) f(z)=h_{p}\left(\alpha_{1}, \ldots, \alpha_{q} ; \beta_{1}, \ldots, \beta_{s} ; z\right) * f(z) .
$$

We observe that, for a function $f(z)$ of the form (1), we have

$$
H_{p}\left(\alpha_{1}, \ldots, \alpha_{q} ; \beta_{1}, \ldots, \beta_{s}\right) f(z)=z^{-p}+\sum_{k=m}^{\infty} \Gamma_{p, q, s}\left(\alpha_{1}\right) a_{k} z^{k} .
$$

where

$$
\Gamma_{p, q, s}\left(\alpha_{1}\right)=\frac{\left(\alpha_{1}\right)_{k+p} \ldots\left(\alpha_{q}\right)_{k+p}}{\left(\beta_{1}\right)_{k+p} \ldots\left(\beta_{s}\right)_{k+p}(k+p) !} .
$$

If, for convenience, we write

$$
H_{p, q, s}\left(\alpha_{1}\right)=H_{p}\left(\alpha_{1}, \ldots, \alpha_{q} ; \beta_{1}, \ldots, \beta_{s}\right),
$$

then one can easily verify from the definition (7) that (see [8])

$$
z\left(H_{p, q, s}\left(\alpha_{1}\right) f(z)\right)^{\prime}=\alpha_{1} H_{p, q, s}\left(\alpha_{1}+1\right) f(z)-\left(\alpha_{1}+p\right) H_{p, q, s}\left(\alpha_{1}\right) f(z) .
$$

For $m=-p+1(p \in \mathbb{N})$, the linear operator $H_{p, q, s}\left(\alpha_{1}\right)$ was investigated recently by Liu and Srivastava [8] and Aouf [2].

In particular, for $\mathrm{q}=2, s=1, \alpha_{1}>0, \beta_{1}>0$ and $\alpha_{2}=1$, we obtain the linear operator

$$
H_{p}\left(\alpha_{1}, 1 ; \beta_{1}\right) f(z)=\ell_{p}\left(\alpha_{1}, \beta_{1}\right) f(z) \quad\left(f \in \Sigma_{p}\right),
$$

which was introduced and studied by Liu and Srivastava [7].

We note that, for any integer $n>-p$ and $f \in \Sigma_{p}$,

$$
H_{p, 2,1}(n+p, 1 ; 1) f(z)=D^{n+p-1} f(z)=\frac{1}{z^{p}(1-z)^{n+p}} * f(z),
$$

where $D^{n+p-1}$ is the differential operator studied by Uralegaddi and Somanatha [16] and Aouf [1].

For functions $f, g \in \Sigma_{p, m}$, we define the linear operator $\mathcal{D}_{\lambda, p}^{n}(f * g): \Sigma_{p, m} \longrightarrow$ $\Sigma_{p, m}\left(\lambda \geq 0 ; p \in \mathbb{N} ; n \in \mathbb{N}_{0}\right)$ by

$$
\mathcal{D}_{\lambda, p}^{0}(f * g)(z)=(f * g)(z),
$$




$$
\begin{aligned}
\mathcal{D}_{\lambda, p}^{1}(f * g)(z) & =\mathcal{D}_{\lambda, p}(f * g)(z) \\
& =(1-\lambda)(f * g)(z)+\lambda z^{-p}\left(z^{p+1}(f * g)(z)\right)^{\prime} \\
& =z^{-p}+\sum_{k=m}^{\infty}[1+\lambda(k+p)] a_{k} b_{k} z^{k}(\lambda \geq 0 ; p \in \mathbb{N}), \\
\mathcal{D}_{\lambda, p}^{2}(f * g)(z) & =\mathcal{D}\left(\mathcal{D}_{\lambda, p}^{1}(f * g)\right)(z) \\
& =(1-\lambda) \mathcal{D}_{\lambda, p}^{1}(f * g)(z)+\lambda z^{-p}\left(z^{p+1} \mathcal{D}_{\lambda, p}^{1}(f * g)(z)\right)^{\prime} \\
& =z^{-p}+\sum_{k=m}^{\infty}[1+\lambda(k+p)]^{2} a_{k} b_{k} z^{k}(\lambda \geq 0 ; p \in \mathbb{N}),
\end{aligned}
$$

and (in general)

$$
\begin{aligned}
\mathcal{D}_{\lambda, p}^{n}(f * g)(z) & =\mathcal{D}\left(\mathcal{D}_{\lambda, p}^{n-1}(f * g)(z)\right)= \\
& =z^{-p}+\sum_{k=m}^{\infty}[1+\lambda(k+p)]^{n} a_{k} b_{k} z^{k}(\lambda \geq 0) .
\end{aligned}
$$

From (13) it is easy to verify that:

$$
z\left(\mathcal{D}_{\lambda, p}^{n}(f * g)(z)\right)^{\prime}=\frac{1}{\lambda} \mathcal{D}_{\lambda, p}^{n+1}(f * g)(z)-\left(p+\frac{1}{\lambda}\right) \mathcal{D}_{\lambda, p}^{n}(f * g)(z)(\lambda>0) .
$$

For $\mathrm{m}=0$ the linear operator $\mathcal{D}_{\lambda, p}^{n}(\mathrm{f} * \mathrm{~g})$ was introduced by Aouf et al. [4].

Making use of the principle of differential subordination as well as the linear operator $D_{\lambda, p}^{n}(f * g)$, we now introduce a subclass of the function class $\Sigma_{p, m}$ as follows:

For fixed parameters $A$ and $B(-1 \leq B<A \leq 1)$, we say that a function $f \in$ $\Sigma_{p, m}$ is in the class $\Sigma_{\lambda, p, m}^{n}(f * g ; A, B)$, if it satisfies the following subordination condition:

$$
-\frac{z^{p+1}\left(\mathcal{D}_{\lambda, p}^{\mathfrak{n}}(f * g)(z)\right)^{\prime}}{p} \prec \frac{1+A z}{1+B z} .
$$

In view of the definition of subordination, (15) is equivalent to the following condition:

$$
\left|\frac{z^{p+1}\left(\mathcal{D}_{\lambda, p}^{n}(f * g)(z)\right)^{\prime}+p}{B z^{p+1}\left(\mathcal{D}_{\lambda, p}^{n}(f * g)(z)\right)^{\prime}+p A}\right|<1 \quad(z \in U) .
$$

For convenience, we write

$$
\Sigma_{\lambda, p}^{n}\left(f * g ; 1-\frac{2 \zeta}{p},-1\right)=\Sigma_{\lambda, p}^{n}(f * g ; \zeta)
$$


where $\Sigma_{\lambda, p}^{n}(f * g ; \zeta)$ denotes the class of functions $f(z) \in \Sigma_{p, m}$ satisfying the following inequality:

$$
\mathfrak{R}\left\{-z^{p+1}\left(\mathcal{D}_{\lambda, p}^{\mathfrak{n}}(\mathrm{f} * \mathrm{~g})(z)\right)^{\prime}\right\}>\zeta \quad(0 \leq \zeta<\mathrm{p} ; z \in \mathrm{U}) .
$$

We note that:

(i) For $b_{k}=\lambda=1$ in (15), the class $\Sigma_{\lambda, p, m}^{n}(f * g ; A, B)$ reduces to the class $\Sigma_{p, m}^{n}(A, B)$ was introduced and studied by Srivastava and Patel [15];

(ii) For $b_{k}=\Gamma_{p, q, s}\left(\alpha_{1}\right)$, where $\Gamma_{p, q, s}\left(\alpha_{1}\right)$ is given by (9), and $n=0$ in (15), we have $\Sigma_{\lambda, p}^{n}(f * g ; A, B)=\Sigma_{p, q, s}^{m}\left(\alpha_{1}, A, B\right)$, where the class $\Sigma_{p, q, s}^{m}\left(\alpha_{1}, A, B\right)$ introduced and studied by Aouf [3];

(iii) For $\mathrm{q}=2, \mathrm{~s}=1, \alpha_{1}=\mathrm{a}>0, \beta_{1}=\mathrm{c}>0$ and $\alpha_{2}=1$, we have $\Sigma_{p, q, s}^{m}\left(\alpha_{1}, A, B\right)=\Sigma_{a, c}(p ; m, A, B)$, where the class $\Sigma_{a, c}(p ; m, A, B)$ was studied by Patel and Cho [13].

\section{Preliminary lemmas}

In order to establish our main results, we need the following lemmas.

Lemma 1 [6]. Let the function $\mathrm{h}$ be analytic and convex (univalent) in $\mathrm{U}$ with $\mathrm{h}(0)=1$. Suppose also that the function $\varphi$ given by

$$
\varphi(z)=1+c_{p+m} z^{p+m}+c_{p+m+1} z^{p+m+1}+\ldots
$$

in analytic in $\mathrm{U}$. If

$$
\varphi(z)+\frac{z \varphi^{\prime}(z)}{\gamma} \prec h(z) \quad(\Re(\gamma) \geq 0 ; \gamma \neq 0),
$$

then

$$
\varphi(z) \prec \psi(z)=\frac{\gamma}{p+m} z^{\frac{-\gamma}{p+m}} \int_{0}^{z} t^{\frac{\gamma}{p+m}-1} h(t) d t \prec h(z),
$$

and $\psi$ is the best dominant. 
For real or complex numbers $\mathrm{a}, \mathrm{b}$ and $\mathrm{c}\left(\mathrm{c} \notin \mathbb{Z}_{0}^{-}\right)$, the Gaussian hypergeometric function is defined by

$$
{ }_{2} \mathrm{~F}_{1}(\mathrm{a}, \mathrm{b} ; \mathrm{c} ; z)=1+\frac{\mathrm{ab}}{\mathrm{c}} \cdot \frac{z}{1 !}+\frac{\mathrm{a}(\mathrm{a}+1) \mathrm{b}(\mathrm{b}+1)}{\mathrm{c}(\mathrm{c}+1)} \cdot \frac{z^{2}}{2 !}+\ldots .
$$

We note that the above series converges absolutely for $z \in U$ and hence represents an analytic function in $\mathrm{U}$ (see, for details [17, Chapter 14]).

Each of the identities (asserted by Lemma 2 below) is well-known (cf., e.g., [17, Chapter 14]).

Lemma 2 [17, Chapter 14]. For real or complex parameters $\mathrm{a}, \mathrm{b}$ and $\mathrm{c}(\mathrm{c} \notin$ $\left.\mathbb{Z}_{0}^{-}\right)$

$$
\int_{0}^{1} t^{b-1}(1-t)^{c-b-1}(1-z t)^{-a} d t=\frac{\Gamma(b) \Gamma(c-b)}{\Gamma(c)}{ }_{2} F_{1}(a, b ; c ; z) \quad(\Re(c)>\Re(b)>0) ;
$$

$$
\begin{gathered}
{ }_{2} \mathrm{~F}_{1}(\mathrm{a}, \mathrm{b} ; \mathrm{c} ; z)=(1-z)^{-\mathrm{a}}{ }_{2} \mathrm{~F}_{1}\left(\mathrm{a}, \mathrm{c}-\mathrm{b} ; \mathrm{c} ; \frac{z}{z-1}\right) \\
{ }_{2} \mathrm{~F}_{1}(\mathrm{a}, \mathrm{b} ; \mathrm{c} ; z)={ }_{2} \mathrm{~F}_{1}(\mathrm{a}, \mathrm{b}-1 ; \mathrm{c} ; z)+\frac{\mathrm{a} z}{\mathrm{c}}{ }_{2} \mathrm{~F}_{1}(\mathrm{a}+1, \mathrm{~b} ; \mathrm{c}+1 ; z)
\end{gathered}
$$

\section{Main results}

Unless otherwise mentioned, we assume throughout this paper that $\lambda, \mu>$ $0, m>-p, p \in \mathbb{N}, n \in \mathbb{N}_{0}$ and $g$ is given by (2).

Theorem 1 Let the function $\mathrm{f}$ defined by (1) satisfying the following subordination condition:

$$
-\frac{(1-\mu) z^{p+1}\left(\mathcal{D}_{\lambda, p}^{n}(f * g)(z)\right)^{\prime}+\mu z^{p+1}\left(\mathcal{D}_{\lambda, p}^{n+1}(f * g)(z)\right)^{\prime}}{p} \prec \frac{1+A z}{1+B z} .
$$

Then

$$
-\frac{z^{\mathfrak{p}+1}\left(\mathcal{D}_{\lambda, p}^{\mathrm{n}}(\mathrm{f} * \mathrm{~g})(z)\right)^{\prime}}{p} \prec \mathcal{G}(z) \prec \frac{1+\mathrm{A} z}{1+\mathrm{B} z},
$$

where the function $\mathcal{G}$ given by

$$
\mathcal{G}(z)= \begin{cases}\frac{A}{B}+\left(1-\frac{A}{B}\right)(1+B z)^{-1}{ }_{2} F_{1}\left(1,1 ; \frac{1}{\lambda \mu(p+m)}+1 ; \frac{B z}{1+B z}\right) & (B \neq 0) \\ 1+\frac{A}{\lambda \mu(p+m)+1} z & (B=0)\end{cases}
$$


is the best dominant of (22). Furthermore,

$$
\mathfrak{R}\left\{-\frac{z^{p+1}\left(\mathcal{D}_{\lambda, p}^{\mathfrak{n}}(\mathrm{f} * \mathrm{~g})(z)\right)^{\prime}}{p}\right\}>\xi \quad(z \in \mathrm{U}),
$$

where

$$
\xi= \begin{cases}\frac{A}{B}+\left(1-\frac{A}{B}\right)(1-B)^{-1}{ }_{2} F_{1}\left(1,1 ; \frac{1}{\lambda \mu(p+m)}+1 ; \frac{B}{B-1}\right) & (B \neq 0) \\ 1-\frac{A}{\lambda \mu(p+m)+1} & (B=0) .\end{cases}
$$

The estimate in (23) is the best possible.

Proof. Consider the function $\varphi$ defined by

$$
\varphi(z)=-\frac{z^{p+1}\left(\mathcal{D}_{\lambda, p}^{\mathfrak{n}}(\mathrm{f} * \mathrm{~g})(z)\right)^{\prime}}{p} \quad(z \in \mathrm{U}) .
$$

Then $\varphi$ is of the form (16) and is analytic in U. Differentiating (24) with respect to $z$ and using (14), we obtain

$$
\begin{gathered}
-\frac{(1-\mu) z^{p+1}\left(\mathcal{D}_{\lambda, p}^{\mathrm{n}}(\mathrm{f} * \mathrm{~g})(z)\right)^{\prime}+\mu z^{\mathrm{p}+1}\left(\mathcal{D}_{\lambda, \mathrm{p}}^{\mathrm{n}+1}(\mathrm{f} * \mathrm{~g})(z)\right)^{\prime}}{p} \\
=\varphi(z)+\lambda \mu z \varphi^{\prime}(z) \prec \frac{1+\mathrm{A} z}{1+\mathrm{Bz}} .
\end{gathered}
$$

Now, by using Lemma 1 for $\beta=\frac{1}{\lambda \mu}$, we obtain

$$
\begin{gathered}
-\frac{z^{p+1}\left(\mathcal{D}_{\lambda, p}^{n}(f * g)(z)\right)^{\prime}}{p} \prec \mathcal{G}(z)=\frac{1}{\lambda \mu(p+m)} z^{-\frac{1}{\lambda \mu(p+m)}} \int_{0}^{z} t^{\frac{1}{\lambda \mu(p+m)}-1}\left(\frac{1+A t}{1+B t}\right) d t \\
\quad= \begin{cases}\frac{A}{B}+\left(1-\frac{A}{B}\right)(1+B z)^{-1}{ }_{2} F_{1}\left(1,1 ; \frac{1}{\lambda \mu(p+m)}+1 ; \frac{B z}{1+B z}\right) & (B \neq 0) \\
1+\frac{A}{\lambda \mu(p+m)+1} z & (B=0),\end{cases}
\end{gathered}
$$

by change of variables followed by the use of the identities (19), (20) and (21) (with $\left.a=1, c=b+1, b=\frac{1}{\lambda \mu(p+m)}\right)$. This proves the assertion (22) of Theorem 1.

Next, in order to prove the assertion (23) of Theorem 1, it suffices to show that

$$
\inf _{|z|<1}\{\mathfrak{R}(\mathcal{G}(z))\}=\mathcal{G}(-1) .
$$


Indeed we have, for $|z| \leq r<1$,

$$
\mathfrak{R}\left(\frac{1+\mathrm{A} z}{1+\mathrm{B} z}\right) \geq \frac{1-\mathrm{Ar}}{1-\mathrm{Br}} .
$$

Upon setting

$$
g(\zeta, z)=\frac{1+A \zeta z}{1+B \zeta z} \text { and } d v(\zeta)=\frac{1}{\lambda \mu(p+m)} \zeta^{\frac{1}{\lambda \mu(p+m)}-1} d \zeta(0 \leq \zeta \leq 1),
$$

which is a positive measure on the closed interval $[0,1]$, we get

$$
\mathcal{G}(z)=\int_{0}^{1} g(\zeta, z) d v(\zeta)
$$

so that

$$
\mathfrak{R}\{\mathcal{G}(z)\} \geq \int_{0}^{1}\left(\frac{1-\mathrm{A} \zeta \mathrm{r}}{1-\mathrm{B} \zeta \mathrm{r}}\right) \mathrm{d} v(\zeta)=\mathcal{G}(-\mathrm{r}) \quad(|z| \leq \mathrm{r}<1) .
$$

Letting $r \rightarrow 1^{-}$in the above inequality, we obtain the assertion (23) of Theorem 1 .

Finally, the estimate in (23) is the best possible as the function $\mathcal{G}$ is the best dominant of (22).

Taking $\mu=1$ in Theorem 1, we obtain the following corollary.

Corollary 1 The following inclusion property holds for the function class $\Sigma_{\lambda, p}^{n}(f * g ; A, B)$ :

$$
\Sigma_{\lambda, p, m}^{n+1}(f * g ; A, B) \subset \Sigma_{\lambda, p, m}^{n}(f * g ; \beta) \subset \Sigma_{\lambda, p, m}^{n}(f * g ; A, B),
$$

where

$$
\beta= \begin{cases}\frac{A}{B}+\left(1-\frac{A}{B}\right)(1-B)^{-1}{ }_{2} F_{1}\left(1,1 ; \frac{1}{\lambda(p+m)}+1 ; \frac{B}{B-1}\right) & (B \neq 0) \\ 1-\frac{A}{\lambda(p+m)+1} & (B=0) .\end{cases}
$$

The result is the best possible.

Taking $\mu=1, A=1-\frac{2 \sigma}{p}(0 \leq \sigma<p)$ and $B=-1$ in Theorem 1 , we obtain the following corollary. 
Corollary 2 The following inclusion property holds for the function class $\Sigma_{\lambda, p, m}^{n}(f * g ; \sigma)$ :

$$
\left.\Sigma_{\lambda, p, m}^{n+1}(f * g ; \sigma) \subset \Sigma_{\lambda, p, m}^{n}(f * g ; \beta)\right) \subset \Sigma_{\lambda, p, m}^{n}(f * g ; \sigma),
$$

where

$$
\beta=\sigma+(p-\sigma)\left\{{ }_{2} F_{1}\left(1,1 ; \frac{1}{\lambda(p+m)}+1 ; \frac{1}{2}\right)-1\right\} .
$$

The result is the best possible.

Theorem 2 If $f \in \Sigma_{\lambda, p, m}^{n}(f * g ; \theta)(0 \leq \theta<p)$, then

$$
\mathfrak{R}\left\{-z^{\mathrm{p}+1}\left[(1-\mu)\left(\mathcal{D}_{\lambda, \mathrm{p}}^{\mathrm{n}}(\mathrm{f} * \mathrm{~g})(z)\right)^{\prime}+\mu\left(\mathcal{D}_{\lambda, \mathrm{p}}^{\mathrm{n}+1}(\mathrm{f} * \mathrm{~g})(z)\right)^{\prime}\right]\right\}>\theta \quad(|z|<\mathrm{R}),
$$

where

$$
R=\left\{\sqrt{1+\lambda^{2} \mu^{2}(p+m)^{2}}-\lambda \mu(p+m)\right\}^{\frac{1}{p+m}} .
$$

The result is the best possible.

Proof. Since $f \in \Sigma_{\lambda, p}^{n}(f * g ; \theta)$, we write

$$
-z^{p+1}\left(\mathcal{D}_{\lambda, p}^{\mathfrak{n}}(f * g)(z)\right)^{\prime}=\theta+(p-\theta) u(z) \quad(z \in \mathbb{U}) .
$$

Then, clearly, $u$ is of the form (16), is analytic in $\mathrm{U}$, and has a positive real part in $\mathrm{U}$. Differentiating (27) with respect to $z$ and using (14), we obtain

$$
-\frac{z^{p+1}\left[(1-\mu)\left(\mathcal{D}_{\lambda, p}^{\mathfrak{n}}(f * g)(z)\right)^{\prime}+\mu\left(\mathcal{D}_{\lambda, p}^{\mathfrak{n}+1}(f * g)(z)\right)^{\prime}\right]+\theta}{p-\theta}=\mathfrak{u}(z)+\lambda \mu z u^{\prime}(z) .
$$

Now, by applying the well-known estimate [5]

$$
\frac{\left|z u^{\prime}(z)\right|}{\mathfrak{R}\{u(z)\}} \leq \frac{2(p+m) r^{p+m}}{1-r^{2(p+m)}}(|z|=r<1)
$$

in (28), we obtain

$$
\mathfrak{R}\left\{-\frac{z^{p+1}\left[(1-\mu)\left(\mathcal{D}_{\lambda, p}^{n}(f * g)(z)\right)^{\prime}+\mu\left(\mathcal{D}_{\lambda, p}^{n+1}(f * g)(z)\right)^{\prime}\right]+\theta}{p-\theta}\right\}
$$




$$
\geq \mathfrak{R}\{u(z)\} \cdot\left(1-\frac{2 \lambda \mu(p+m) r^{p+m}}{\left(1-r^{2(p+m)}\right)}\right)
$$

It is easily seen that the right-hand side of (29) is positive provided that $r<R$, where $R$ is given as in Theorem 2. This proves the assertion (26) of Theorem 2 .

In order to show that the bound $\mathrm{R}$ is the best possible, we consider the function $f \in \Sigma_{p, m}$ defined by

$$
-z^{p+1}\left(\mathcal{D}_{\lambda, p}^{n}(f * g)(z)\right)^{\prime}=\theta+(p-\theta) \frac{1+z^{p+m}}{1-z^{p+m}} \quad(0 \leq \theta<p ; p \in \mathbb{N} ; z \in U) .
$$

Noting that

$$
\begin{aligned}
& -\frac{z^{p+1}\left[(1-\mu)\left(\mathcal{D}_{\lambda, p}^{\mathfrak{n}}(f * g)(z)\right)^{\prime}+\mu\left(\mathcal{D}_{\lambda, p}^{n+1}(f * g)(z)\right)^{\prime}\right]+\theta}{p-\theta} \\
= & \frac{1-z^{2(p+m)}+2 \lambda \mu(p+m) z^{p+m}}{\alpha_{1}\left(1-z^{p+m}\right)^{2}}=0
\end{aligned}
$$

for $z=R^{\frac{1}{p+m}} \exp \left(\frac{i \pi}{p+m}\right)$, we complete the proof of Theorem 2 .

Putting $\mu=1$ in Theorem 2, we obtain the following result.

Corollary 3 If $f \in \Sigma_{\lambda, p, m}^{n}(f * g ; \theta)(0 \leq \theta<p ; p \in \mathbb{N})$, then $f$ satisfies the condition of $\Sigma_{\lambda, \mathfrak{p}, \mathrm{m}}^{\mathfrak{n}+1}(\mathrm{f} * \mathrm{~g} ; \theta)$ for $|z|<\mathrm{R}^{*}$, where

$$
R^{*}=\left\{\sqrt{1+\lambda^{2}(p+m)^{2}}-\lambda(p+m)\right\}^{\frac{1}{p+m}} .
$$

The result is the best possible.

Theorem 3 Let $\mathrm{f} \in \Sigma_{\lambda, \mathrm{p}, \mathrm{m}}^{\mathfrak{n}}(\mathrm{f} * \mathrm{~g} ; \mathrm{A}, \mathrm{B})$ and let

$$
F_{\delta, p}(f)(z)=\frac{\delta}{z^{\delta+p}} \int_{0}^{z} t^{\delta+p-1} f(t) d t \quad(\delta>0 ; z \in U)
$$

Then

$$
-\frac{z^{p+1}\left(\mathcal{D}_{\lambda, p}^{\mathfrak{n}}\left(\mathrm{F}_{\delta, p}(f) * g\right)(z)\right)^{\prime}}{p} \prec \Phi(z) \prec \frac{1+A z}{1+\mathrm{Bz}},
$$


where the function $\Phi$ given by

$$
\Phi(z)= \begin{cases}\frac{\mathrm{A}}{\mathrm{B}}+\left(1-\frac{\mathrm{A}}{\mathrm{B}}\right)(1+\mathrm{B} z)^{-1}{ }_{2} \mathrm{~F}_{1}\left(1,1 ; \frac{\delta}{\mathrm{p}+\mathrm{m}}+1 ; \frac{\mathrm{B} z}{\mathrm{~B} z+1}\right) & (\mathrm{B} \neq 0) \\ 1+\frac{\delta}{\delta+\mathrm{p}+\mathrm{m}} \mathrm{A} z & (\mathrm{~B}=0),\end{cases}
$$

is the best dominant of (31). Furthermore,

$$
\mathfrak{R}\left\{-\frac{z^{p+1}\left(\mathcal{D}_{\lambda, p}^{\mathfrak{n}}\left(\mathrm{F}_{\delta, p}(\mathrm{f}) * \mathrm{~g}\right)(z)\right)^{\prime}}{p}\right\}>\xi^{*} \quad(z \in \mathrm{U}),
$$

where

$$
\xi^{*}= \begin{cases}\frac{A}{B}+\left(1-\frac{A}{B}\right)(1-B)^{-1}{ }_{2} F_{1}\left(1,1 ; \frac{\delta}{p+m}+1 ; \frac{B}{B-1}\right) & (B \neq 0) \\ 1-\frac{\delta}{\delta+p+m} A & (B=0) .\end{cases}
$$

The result is the best possible.

Proof. Defining the function $\varphi$ by

$$
\varphi(z)=-\frac{z^{p+1}\left(\mathcal{D}_{\lambda, p}^{\mathfrak{n}}\left(F_{\delta, p}(f) * g\right)(z)\right)^{\prime}}{p}(z \in \mathrm{U}),
$$

we note that $\varphi$ is of the form (16) and is analytic in U. Using the following operator identity:

$$
z\left(\mathcal{D}_{\lambda, p}^{n}\left(F_{\delta, p}(f) * g\right)(z)\right)^{\prime}=\delta \mathcal{D}_{\lambda, p}^{n}(f * g)(z)-(\delta+p) \mathcal{D}_{\lambda, p}^{n}\left(F_{\delta, p}(f) * g\right)(z)
$$

in (33) and differentiating the resulting equation with respect to $z$, we find that

$$
-\frac{z^{p+1}\left(\mathcal{D}_{\lambda, p}^{n}(f * g)(z)\right)^{\prime}}{p}=\varphi(z)+\frac{z \varphi^{\prime}(z)}{\delta} \prec \frac{1+A z}{1+B z} .
$$

Now the remaining part of Theorem 3 follows by employing the techniques that we used in proving Theorem 1 above.

Remark 1 By observing that

$$
z^{p+1}\left(\mathcal{D}_{\lambda, p}^{n}\left(F_{\delta, p}(f) * g\right)(z)\right)^{\prime}=\frac{\delta}{z^{\delta}} \int_{0}^{z} t^{\delta+p}\left(\mathcal{D}_{\lambda, p}^{n}(f * g)(t)\right)^{\prime} d t \quad\left(f \in \Sigma_{p, m} ; z \in U\right) .
$$


If $\delta>0$ and $f \in \Sigma_{\lambda, p, m}^{n}(f * g ; A, B)$, then

$$
\mathfrak{R}\left\{-\frac{\delta}{p z^{\delta}} \int_{0}^{z} t^{\delta+p}\left(\mathcal{D}_{\lambda, p}^{n}(f * g)(t)\right)^{\prime} d t\right\}>\xi^{*}(z \in U),
$$

where $\xi^{*}$ is given as in Theorem 3.

In view of (35), Theorem 3 for $\mathrm{A}=1-\frac{2 \theta}{\mathrm{p}}(0 \leq \theta<\mathrm{p} ; \mathrm{p} \in \mathbb{N})$ and $\mathrm{B}=-1$ yields

Corollary 4 If $\delta>0$ and if $\mathrm{f} \in \Sigma_{p, m}$ satisfies the following inequality:

$$
\mathfrak{R}\left\{-z^{p+1}\left(\mathcal{D}_{\lambda, p}^{\mathfrak{n}}(\mathrm{f} * \mathrm{~g})(z)\right)^{\prime}\right\}>\theta \quad(0 \leq \theta<\mathrm{p} ; \mathrm{p} \in \mathbb{N} ; z \in \mathrm{U}),
$$

then

$\mathfrak{R}\left\{\frac{-\delta}{z^{\delta}} \int_{0}^{z}\left(\mathcal{D}_{\lambda, p}^{n}(f * g)(t)\right)^{\prime} d t\right\}>\theta+(p-\theta)\left[{ }_{2} \mathrm{~F}_{1}\left(1,1 ; \frac{\delta}{p+m}+1 ; \frac{1}{2}\right)-1\right](z \in \mathrm{U})$.

The result is the best possible.

Theorem 4 Let $\mathrm{f} \in \Sigma_{\mathrm{p}, \mathrm{m}}$. Suppose also that $\mathrm{h} \in \Sigma_{\mathrm{p}, \mathrm{m}}$ satisfies the following inequality:

$$
\mathfrak{R}\left\{z^{\mathrm{p}}\left(\mathcal{D}_{\lambda, p}^{\mathrm{n}}(\mathrm{h} * \mathrm{~g})(z)\right)\right\}>0 \quad(z \in \mathrm{U}) .
$$

If

$$
\left|\frac{\mathcal{D}_{\lambda, p}^{\mathrm{n}}(\mathrm{f} * \mathrm{~g})(z)}{\mathcal{D}_{\lambda, p}^{\mathrm{n}}(\mathrm{h} * \mathrm{~g})(z)}-1\right|<1 \quad(z \in \mathrm{U})
$$

then

$$
\mathfrak{R}\left\{-\frac{z\left(\mathcal{D}_{\lambda, p}^{n}(f * g)(z)\right)^{\prime}}{\mathcal{D}_{\lambda, p}^{n}(f * g)(z)}\right\}>0 \quad\left(|z|<R_{0}\right),
$$

where

$$
R_{0}=\left[\frac{\sqrt{9(p+m)^{2}+4 p(2 p+m)}-3(p+m)}{2(2 p+m)}\right]^{\frac{1}{p+m}} .
$$

Proof. Letting

$$
w(z)=\frac{\mathcal{D}_{\lambda, p}^{n}(f * g)(z)}{\mathcal{D}_{\lambda, p}^{n}(h * g)(z)}-1=t_{p+m} z^{p+m}+t_{p+m+1} z^{p+m+1}+\ldots,
$$


we note that $w$ is analytic in $\mathrm{U}$, with $w(0)=0$ and $|w(z)| \leq|z|^{p+m}(z \in \mathrm{U})$. Then, by applying the familiar Schwarz's lemma [12], we obtain

$$
w(z)=z^{p+m} \Psi(z),
$$

where the functions $\Psi$ is analytic in $\mathrm{U}$ and $|\Psi(z)| \leq 1(z \in \mathrm{U})$. Therefore, (36) leads us to

$$
\mathcal{D}_{\lambda, p}^{n}(f * g)(z)=\mathcal{D}_{\lambda, p}^{n}(h * g)(z)\left(1+z^{p+m} \Psi(z)\right) \quad(z \in \mathrm{U}) .
$$

Differentiating (37) logarithmically with respect to $z$, we obtain

$$
\frac{z\left(\mathcal{D}_{\lambda, p}^{\mathrm{n}}(\mathrm{f} * \mathrm{~g})(z)\right)^{\prime}}{\mathcal{D}_{\lambda, p}^{\mathrm{n}}(\mathrm{f} * \mathrm{~g})(z)}=\frac{z\left(\mathcal{D}_{\lambda, \mathrm{p}}^{\mathrm{n}}(\mathrm{h} * \mathrm{~g})(z)\right)^{\prime}}{\mathcal{D}_{\lambda, p}^{\mathrm{n}}(\mathrm{h} * \mathrm{~g})(z)}+\frac{z^{\mathrm{p}+\mathrm{m}}\left\{(\mathrm{p}+\mathrm{m}) \Psi(z)+z \Psi^{\prime}(z)\right\}}{1+z^{p+m} \Psi(z)} .
$$

Putting $\varphi(z)=z^{\mathfrak{p}} \mathcal{D}_{\lambda, p}^{\mathfrak{n}}(\mathrm{h} * \mathrm{~g})(z)$, we see that the function $\varphi$ is of the form (16), is analytic in $\mathrm{U}, \mathfrak{R}\{\varphi(z)\}>0(z \in \mathrm{U})$ and

$$
\frac{z\left(\mathcal{D}_{\lambda, p}^{\mathrm{n}}(\mathrm{h} * \mathrm{~g})(z)\right)^{\prime}}{\mathcal{D}_{\lambda, \mathrm{p}}^{\mathrm{n}}(\mathrm{h} * \mathrm{~g})(z)}=\frac{z \varphi^{\prime}(z)}{\varphi(z)}-p
$$

so that we find from (38) that

$$
\begin{aligned}
& \Re\left\{-\frac{z\left(\mathcal{D}_{\lambda, p}^{n}(\mathrm{f} * \mathrm{~g})(z)\right)^{\prime}}{\mathcal{D}_{\lambda, p}^{\mathrm{n}}(\mathrm{f} * \mathrm{~g})(z)}\right\} \geq p \\
& \quad-\left|\frac{z \varphi^{\prime}(z)}{\varphi(z)}\right|-\left|\frac{z^{p+m}\left\{(p+m) \Psi(z)+z \Psi^{\prime}(z)\right\}}{1+z^{p+m} \Psi(z)}\right| \quad(z \in \mathrm{U}) .
\end{aligned}
$$

Now, by using the following known estimates [9]:

$$
\left|\frac{\varphi^{\prime}(z)}{\varphi(z)}\right| \leq \frac{2(p+m) r^{p+m-1}}{1-r^{2(p+m)}} \quad(|z|=r<1)
$$

and

$$
\left|\frac{(p+m) \Psi(z)+z \Psi^{\prime}(z)}{1+z^{p+m} \Psi(z)}\right| \leq \frac{(p+m)}{1-r^{p+m}} \quad(|z|=r<1)
$$

in (39), we obtain

$\mathfrak{R}\left\{-\frac{z\left(\mathcal{D}_{\lambda, p}^{n}(f * g)(z)\right)^{\prime}}{\mathcal{D}_{\lambda, p}^{n}(f * g)(z)}\right\} \geq \frac{p-3(p+m) r^{p+m}-(2 p+m) r^{2(p+m)}}{1-r^{2(p+m)}}(|z|=r<1)$, 
which is certainly positive, provided that $r<R_{0}, R_{0}$ being given as in Theorem 4 .

Theorem 5 If $\mathrm{f} \in \Sigma_{\mathrm{p}, \mathrm{m}}$ satisfies the following subordination condition:

$$
(1-\mu) z^{p} \mathcal{D}_{\lambda, p}^{n}(f * g)(z)+\mu z^{p} \mathcal{D}_{\lambda, p}^{n+1}(f * g)(z) \prec \frac{1+A z}{1+B z},
$$

then

$$
\mathfrak{R}\left\{z^{\mathfrak{p}} \mathcal{D}_{\lambda, p}^{\mathfrak{n}}(\mathrm{f} * \mathrm{~g})(z)\right\}^{\frac{1}{\mathrm{~d}}}>\xi^{\frac{1}{\mathrm{~d}}} \quad(\mathrm{~d} \in \mathbb{N} ; z \in \mathrm{U}),
$$

where $\xi$ is given as in Theorem 1. The result is the best possible.

Proof. Defining the function $\varphi$ by

$$
\varphi(z)=z^{\mathfrak{p}} \mathcal{D}_{\lambda, p}^{\mathfrak{n}}(\mathrm{f} * \mathrm{~g})(z) \quad\left(\mathrm{f} \in \Sigma_{\mathrm{p}, \mathrm{m}} ; z \in \mathrm{U}\right),
$$

we see that the function $\varphi$ is of the form (16) and is analytic in U. Differentiating (40) with respect to $z$ and using the identity (14), we obtain

$$
(1-\mu) z^{p} \mathcal{D}_{\lambda, p}^{n}(f * g)(z)+\mu z^{p} \mathcal{D}_{\lambda, p}^{n+1}(f * g)(z)=\varphi(z)+\lambda \mu z \varphi^{\prime}(z) \prec \frac{1+A z}{1+B z} .
$$

Now, by following the lines of the proof of Theorem 1 mutatis mutandis, and using the elementary inequality:

$$
\mathfrak{R}\left(w^{\frac{1}{d}}\right) \geq(\mathfrak{R} w)^{\frac{1}{d}}(\mathfrak{R}(w)>0 ; d \in \mathbb{N}),
$$

we arrive at the result asserted by Theorem 5 .

Remark 2 (i) Taking $b_{k}=\lambda=1$ in the above results, we obtain the results obtained by Srivastava and Patel [15];

(ii) Taking $\mathrm{b}_{\mathrm{k}}=\Gamma_{\mathrm{p}, \mathrm{q}, \mathrm{s}}\left(\alpha_{1}\right)$, where $\Gamma_{\mathrm{p}, \mathrm{q}, \mathrm{s}}\left(\alpha_{1}\right)$ is given by (9), and $\mathrm{n}=0$ in the above results, we obtain the results obtained by Aouf [3].

\section{References}

[1] M. K. Aouf, New criteria for multivalent meromorphic starlike functions of order alpha, Proc. Japan Acad., 69 Ser. A (1993), 66-70. 
[2] M. K. Aouf, Certain subclasses of meromorphically multivalent functions associated with generalized hypergeometric function, Comput. Math. Appl., 55 (3) (2008), 494-509.

[3] M. K. Aouf, Applications of differential subodination to certain subclasses of meromorphically multivalent functions associated with generalized hypergeometric function, Indian J. Math., 52 (2) (2010), 357-380.

[4] M. K. Aouf, A. Shamandy, A. O. Mostafa, S. M. Madian, Properties of some families of meromorphic p-valent functions involving certain differential operator, Acta Univ. Apulensis, 20 (2009), 7-16.

[5] S. D. Bernardi, New distortion theorems for functions of positive real part and applications to the partial sums of univalent convex functions, Proc. Amer. Math. Soc., 45 (1) (1974), 113-118.

[6] D. J. Hallenbeck, St. Ruscheweyh, Subordination by convex functions, Proc. Amer. Math. Soc., 52 (1975), 191-195.

[7] J.-L. Liu, H. M. Srivastava, A linear operator and associated families of meromorphically multivalent functions, J. Math. Anal. Appl., 259 (2000), $566-581$.

[8] J.-L. Liu, H. M. Srivastava, Classes of meromorphically multivalent functions associated with the generalized hypergeometric function, Math. Comput. Modelling, 39 (2004), 21-34.

[9] T. H. MacGregor, Radius of univalence of certain analytic functions, Proc. Amer. Math. Soc., 14 (1963), 514-520.

[10] S. S. Miller, P. T. Mocanu, Differential subordinations and univalent functions, Michigan Math. J., 28 (1981), 157-171.

[11] S. S. Miller, P. T. Mocanu, Differential subordinations: Theory and applications, Series on Monographs and Texbooks in Pure and Applied Mathematics, 225, Marcel Dekker, New York and Basel, 2000.

[12] Z. Nehari, Conformal mapping, McGraw-Hill, New York, 1952.

[13] J. Patel, N. E. Cho, Certain subclasses of meromorphically p-valent functions involving a linear operator, Math. Sci. Res. J., 7 (4) (2003), 117-128. 
[14] H. M. Srivastava, P. W. Karlsson, Multiple Gaussian hypergeometric series, Halsted Press, Ellis Horwood Limited, Chichester, John Wiley and Sons, New York, Chichester, Brisbane, Toronto, 1985.

[15] H. M. Srivastava, J. Patel, Applications of differential subodination to certain subclasses of meromorphically multivalent functions, J. Inequal. Pure Appl. Math., 6 (3) (2005), Art. 88, pp. 1-15.

[16] B. A. Uralegaddi, C. Somanatha, Certain classes of meromorphic multivalent functions, Tamkang J. Math., 23 (1992), 223-231.

[17] E. T. Whittaker and G. N. Watson, A course on modern analysis : An introduction to the general theory of infinite processes and of analytic functions; with an account of the principal transcendental functions, Fourth Edition (Reprinted), Cambridge University Press, Cambridge, 1927.

Received: 9 May 2013 